\title{
Memory problems after epilepsy surgery
}

Stephen L. Holliday, PhD; and Robin L. Brey, MD

Surgery is performed on some patients with epilepsy. This treatment is usually considered when standard medical treatment is not effective in controlling seizures, when the area of the brain from which the seizures are coming (also called "seizure focus") can be identified, and when seizures are restricted to only one side of the brain. Approximately $80 \%$ of adult epilepsy patients who undergo this surgery have significant improvement in seizure control. More information about epilepsy surgery can be found on the next page.

\section{Memory Problems After Epilepsy Surgery}

One of the side effects of epilepsy surgery can be memory problems. From prior studies, the risk of this seems greater when the surgery is performed on the left side of the brain. It is unclear whether these memory problems after epilepsy surgery are any worse than in patients with poorly controlled seizures who do not have epilepsy surgery. In this issue of Neurology, Rausch et al. report a study of memory function 9 to 19 years after epilepsy surgery in patients who had left vs right-sided surgery. Both groups were compared to epilepsy patients who had similar seizure disorders and were followed for the same time period, but did not have surgery.

There were 21 patients who had epilepsy surgery on the left side of the brain, 23 who had epilepsy surgery on the right side of the brain, and 8 patients who did not have surgery. Memory function and seizure control was measured in each group 1 year after the surgery, or 1 year after the surgical evalua- tion in the group who did not have epilepsy surgery. All groups were evaluated again at an average of 12 years later.

\section{Patients With Epilepsy Surgery on the Left Side of the Brain Have More Memory Problems}

The 1 st year after surgery, $88 \%$ of the nonsurgery patients and $50 \%$ of the surgery patients reported having at least one seizure. In the year before the long-term follow-up evaluation, $88 \%$ of the nonsurgery and $20.5 \%$ of the surgery patients reported having at least one seizure. At long-term follow-up, all nonsurgery patients and $71 \%$ of the surgery patients were taking antiseizure medications. Before surgery, the patients with a left-sided seizure focus had mild memory problems on a test where they had to remember pairs of words. One year later, these verbal memory problems had worsened in the patients with left-sided epilepsy surgery, whereas the patients with right-sided epilepsy surgery had improvement in verbal memory function. The nonsurgical group had not changed. At the time of long-term follow-up, the nonsurgery patients and the patients with left-sided epilepsy surgery had more memory problems than the patients with right-sided epilepsy surgery, but all groups had some decline in memory functioning. None had improvement in any memory tests at long-term followup. The numbers of seizures and memory problems that patients were experiencing at long-term follow-up were both associated with patients' reports of a lower quality of life.

\section{What Are the Memory Problems in Epilepsy Patients Due To?}

This study shows that severe epilepsy itself can result in memory decline over time, with or without surgical treatment. However, it also demonstrated that surgical patients with left-sided brain lesions had more verbal memory decline than those who had right-sided lesions. The reason for this may be that an area of the brain that is important for memory functions, the hippocampus, appears to shrink in the first 3 months after surgery. The left hippocampus is important for verbal memory functioning. The continued worsening of memory functioning in all groups may be due to an acceleration of the normal aging process that is caused by hippocampal damage due to postsurgical shrinkage or continued seizures. Another possibility is that antiepileptic medications used in all groups could affect memory function, but the study did not have a large enough number of patients to assess this.

In summary, this study shows that epilepsy surgery of either side of the brain improves seizure control. This leads to a decreased need for antiepileptic medication and a better quality of life for these patients. All epilepsy patients, whether they undergo surgery or not, have some decline in memory function. It is important to note that the patients in this study most likely had severe epilepsy because they were referred for surgery. We do not know whether milder forms of epilepsy lead to memory decline. 
$1 \%$ of the US population.

Epilepsy is a chronic neurologic disorder characterized by recurrent, unprovoked seizures. A seizure is a self-limited spell in which a part of the brain is uncontrollably activated. Patients with epilepsy are usually treated with medication. For many patients, this treatment controls their seizures. Unfortunately, some patients will continue to have seizures despite taking their medications. When this happens, brain surgery is another treatment option.

\section{Surgery as a Treatment for Epilepsy}

There are as many as 100,000 people with epilepsy in the US who could benefit from epilepsy surgery. The effectiveness of epilepsy surgery depends on the cause of the epilepsy and the careful selection of patients who could benefit the most (table).

Table When is epilepsy surgery considered as a treatment for epilepsy?

The person has no other medical problem that would make them unsuitable for this type of surgery.

Antiepileptic drug treatment has been tried but has been proved to be unsuitable or unsuccessful.

The seizures can be seen to be arising from one localized area of the brain.

The person's ability to function normally would not be affected by removing this part of the brain.

The irregular part of the brain is accessible to the surgeon and can be removed without causing further damage to any other part of the brain.

The areas of the brain responsible for speech, sight, movement, or hearing are not close to the part of the brain to be removed.

The person is thought to have a very good chance of becoming seizure free after surgery.

\section{How Do We Tell Which \\ Epilepsy Patients May \\ Benefit Most From Epilepsy Surgery?}

The choice of who should have epilepsy surgery is complicated and requires many specialized tests.

The most important piece of information that doctors need to know before removing the epileptic part of the brain is its exact location. This is often determined using video-EEG monitoring. Electrodes are placed on the scalp using a type of glue. The brain wave activity is recorded using these electrodes. The patient is also videotaped so that the brainwave activity during the exact time of the seizure can be studied. This often allows doctors to see the place in the brain that the epilepsy is coming from during the time of the seizure.

A WADA test is usually performed. This is a test that doctors use to determine where the language abilities are located in the patient's brain and whether their memory will be altered by the surgery. This test involves placing a small catheter in a blood vessel in the thigh and moving the catheter up to the neck within the blood vessels using $\mathrm{x}$-ray guidance. A medicine is then injected into the blood vessels of the brain to put part of the brain to sleep for a few minutes. The doctors will then do tests to see how memory and language abilities are affected. This test provides very important information that helps the epilepsy surgeon plan the safest possible surgery for each individual patient.

A brain imaging study called a MRI is done to look at abnormal areas of the brain. Neuropsychological tests are done to evaluate memory and thinking function.

\section{What Are the Risks Involved in Epilepsy Surgery?}

As with most surgeries, there is a small risk of infection or a hemorrhage, but this is usually less than $1 \%$. Usually, these complications can be treated and do not cause any permanent problems. Neurosurgeons now operate with microscopes to visualize small anatomical details more accurately. Computers are routinely used to guide operations. Instruments have evolved to minimize trauma to the normal brain not being removed. Because of this, there is a very small risk of weakness in your arm and leg, or of having wordfinding difficulties after surgery. As the article on page 1 of this Neurology Patient Page reports, some memory difficulties also occur, especially after epilepsy surgery involving the left side of the brain. However, information from the WADA test is used to determine the chances of this occurring for an individual patient.

\section{What Happens During the Surgery?}

The area of abnormal brain that the epilepsy is coming from identified in the presurgical evaluation is removed. The exact details are different for each patient. A discussion covering these details occurs between the patient and their family, the neurologist and the neurosurgeon who will be performing the epilepsy surgery.

\section{How Long Does It Take to Recover From Epilepsy Surgery?}

Most patients stay in the intensive care unit overnight and then stay in a regular floor bed for 3 or 4 days. Seizure medications are usually continued just as they were before surgery. Eventually, seizure medications may be decreased or 
stopped completely, but this is very different for each patient and should be discussed with the doctors.

\section{What Are the Chances of Epilepsy Surgery Curing or Improving Epilepsy?}

This depends on the cause of the epilepsy and can be as high as $80 \%$ in carefully selected adults and $40-60 \%$ in children. In addition, there is a good chance that even if all seizures do not stop after brain surgery, the seizures will decrease in frequency and fewer seizure medications may be needed.
For more Information

Epilepsy Foundation of America: www.efa.org

American Academy of Neurology: www.aan.com 


\title{
Neurology
}

\author{
Memory problems after epilepsy surgery \\ Stephen L. Holliday and Robin L. Brey \\ Neurology 2003;60;E3-E5 \\ DOI 10.1212/WNL.60.6.E3
}

This information is current as of March 25, 2003

\section{Updated Information \&}

Services

Permissions \& Licensing

Reprints including high resolution figures, can be found at:

http://n.neurology.org/content/60/6/E3.full

Information about reproducing this article in parts (figures,tables) or in its entirety can be found online at:

http://www.neurology.org/about/about_the_journal\#permissions

Information about ordering reprints can be found online:

http://n.neurology.org/subscribers/advertise

Neurology ${ }^{\circledR}$ is the official journal of the American Academy of Neurology. Published continuously since 1951, it is now a weekly with 48 issues per year. Copyright . All rights reserved. Print ISSN: 0028-3878. Online ISSN: 1526-632X.

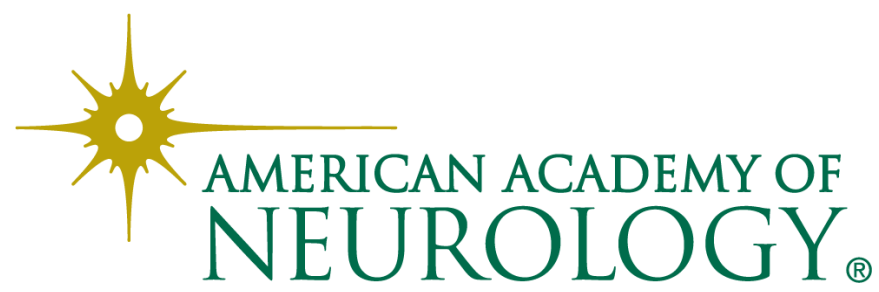

